\title{
Comunicación
}

\section{Primer registro de enfermedad fúngica en una serpiente sudamericana: características clínicas y patológicas}

\author{
First REPORT OF FUNGaL DISEASE IN A SOUTH AMERICAN SNAKE
}

\author{
María Lucía Bustos ${ }^{1}$, Matías Nicolás Sánchez², María Elisa Peichoto², \\ Gladys Pamela Teibler ${ }^{3,4}$
}

\section{RESUMEN}

La Enfermedad Fúngica de las Serpientes (EFS) ha sido reportada en varias especies de serpientes cascabel y de colúbridos de Estados Unidos de América y Europa, pero no en América del Sur. Un espécimen del colúbrido sudamericano Xenodon merremii (conocido popularmente como falsa yarará) arribó al Servicio de Emergencia del Hospital de Clínicas de la Facultad de Ciencias Veterinarias de la Universidad Nacional del Nordeste (Argentina), presentando una solución de continuidad costrosa en la piel, y otra lesión consistente en decoloración y pérdida de brillo de las escamas. El ejemplar falleció a los 16 días de iniciado el tratamiento. A la necropsia, se observaron lesiones en el parénquima hepático, mientras que el pulmón no evidenció lesiones macroscópicas apreciables. Las lesiones encontradas fueron compatibles con EFS.

Palabras clave: Colubridae; dermatitis; ofidio; neumonía micótica

\section{Abstract}

The Snake Fungal Disease (SFD) has been reported in several species of rattlesnakes and colubrid snakes in the United States of America and Europe, but not in South America. A specimen of the South American colubrid (Xenodon merremii, commonly known as false yarara) was presented at the Servicio de Emergencias del Hospital de

\footnotetext{
${ }^{1}$ Instituto Nacional de Medicina Tropical (INMeT), Puerto Iguazú, Misiones, Argentina

${ }^{2}$ Consejo Nacional de Investigaciones Científicas y Técnicas (CONICET); INMeT, Puerto Iguazú, Misiones, Argentina

${ }^{3}$ Cátedra de Farmacología y Toxicología, Facultad de Ciencias Veterinarias, Universidad Nacional del Nordeste (UNNE), Corrientes, Argentina

${ }^{4}$ E-mail: pteibler@vet.unne.edu.ar
}

Recibido: 21 de diciembre de 2017

Aceptado para publicación: 18 de junio de 2018 
Clínicas de la Facultad de Ciencias Veterinarias, Universidad Nacional del Nordeste (Argentina), presenting a crusty open wound in the skin, and another injury consisting of discolored and dull scales. The specimen died 16 days after starting the treatment. At necropsy, lesions in the hepatic parenchyma were observed, whereas the lung showed no appreciable macroscopic lesions. The lesions found in this case were compatible with SFD.

Key words: Colubridae; dermatitis; snake; mycotic pneumonia

\section{INTRODUCCIÓN}

La Enfermedad Fúngica de las Serpientes (EFS) es un proceso patológico que se caracteriza por lesiones dérmicas en el cuello y la cabeza que varían desde pústulas o nódulos hasta hinchazón severa de la piel (Allender et al., 2011; Sleeman, 2013); sin embargo, en algunos casos las lesiones abarcan otras regiones del cuerpo (Sleeman, 2013). La EFS afecta mayormente a serpientes del género Crotalus (cascabel) y a varios géneros de colúbridos (Allender et al., 2015 b). La enfermedad es causada por patógenos oportunistas que infectan animales que tienen el sistema inmune deprimido (Allender et al., 2015a), especialmente por Ophidiomyces ophiodiicola, hongo saprófito que posee una extraordinaria capacidad de adaptarse a diferentes ambientes, presentando una alta virulencia (Allender et al., 2015b).

Allender (2017) reportó un brote de micosis en Florida, EEUU, en el año 2000, en 42 ejemplares de cascabel pigmea (Sistrurus miliarius), en una culebra rayada (Thamnophis sp) y en una serpiente de cinta común (Thamnophis sauritus), cuyos síntomas fueron compatibles con la EFS; sin embargo, en esa ocasión no se realizó la identificación del agente causal. En 2006, Allender (2017) reportó en New Hampshire, EEUU, la presencia de lesiones dérmicas en varias partes del cuerpo de una población de cascabel de los bosques (Crotalus horridus), siendo estas lesiones similares a las descritas en la EFS. En 2008 se realizó por primera vez el diagnóstico confirmatorio de $O$. ophiodiicola como principal agente causal de EFS en una población de víboras de cascabel pigmea norteña (Sistrurus catenatus) en Carlyle Lake, Illinois, EEUU, utilizando la técnica de la Reacción en Cadena de la Polimerasa (PCR) convencional (Allender et al., 2015c). Estudios experimentales demostraron que $O$. ophiodiicola puede actuar como patógeno primario y causar la muerte del animal sin participación de otros agentes patógenos (Allender et al., 2015a).

Si bien la distribución global de la EFS es desconocida, hasta ahora solo ha sido reportada en Norteamérica y en Europa (Franklinos et al., 2017), pero es posible su diseminación a otros ecosistemas por medio del tráfico o traslado de animales. La infección puede ocurrir por contacto directo con serpientes infectadas, o porque el agente causal se encuentra presente en el ambiente; además, es posible la transmisión por medio de equipos, vestimenta y calzados contaminados y usados para el manejo de las serpientes (Allender et al., 2015c).

Las lesiones observadas en piel consisten en dermatitis, costras de tonalidad amarillenta a marrón, despigmentación o pérdida de brillo de las escamas, presencia de ampollas, nódulos subcutáneos e incluso heridas abiertas (Allender et al., 2015c). A nivel sistémico, las manifestaciones de la EFS son variables e incluyen neumonía granulomatosa, hepatitis, esplenitis, nefritis e infecciones oculares (Vissiennon et al., 1999; Rajeev et al., 2009; Sleeman, 2013; Dolinski et al., 2014). 
Teniendo en cuenta la importancia de contribuir con el conocimiento de la EFS, enfermedad emergente de preocupación para la conservación de especies de serpientes, se describe en el presente trabajo el caso de un espécimen del colúbrido sudamericano Xenodon merremii (falsa yarará) que arribó al Servicio de Emergencia del Hospital de Clínicas de la Facultad de Ciencias Veterinarias (FCV) de la Universidad Nacional del Nordeste (UNNE) con signos clínicos compatibles con la EFS.

\section{Caso Clínico}

Un ejemplar de la familia Colubridae, proveniente del municipio de Riachuelo $\left(27^{\circ}\right.$ $34^{\prime} 47.2^{\prime \prime} \mathrm{S}, 58^{\circ} 45^{\prime} 28.5^{\prime \prime} \mathrm{W}$ ), provincia de Corrientes (Argentina), arribó al Servicio de Emergencia del Hospital de Clínicas de la FCV-UNNE el 7 de septiembre de 2016, siendo el motivo de la consulta la presencia de una lesión ulcerativa en el tercio medio del cuerpo. La identificación del espécimen se realizó según Giraudo (2004).

La evaluación clínica se realizó según la modalidad descripta por Varela (2002). E1 sexado se realizó mediante el uso de un catéter (Varela, 2002). La restricción del paciente se realizó por medios físicos. A través de la inspección se determinó el Estado de Desarrollo Biológico (EDB) y se midió la longitud hocico-cloaca (LHC o SVL en inglés) mediante el uso de la técnica de análisis morfométricos (Varela, 2002). El ejemplar correspondió a un macho adulto $(57 \mathrm{~cm}$ de largo, $60 \mathrm{~g}$ de peso) de Xenodon merremii (nombre común: falsa yarará o culebra sapera) (Figura 1).

Se constató actividad estática y dinámica normal, temperamento linfático, estado general bueno, y actividad motriz aletargada. Presentó una deshidratación moderada, evidenciada por sequedad de mucosa oral y pérdida de elasticidad de la piel. Se observó una lesión profunda en piel de la porción me-

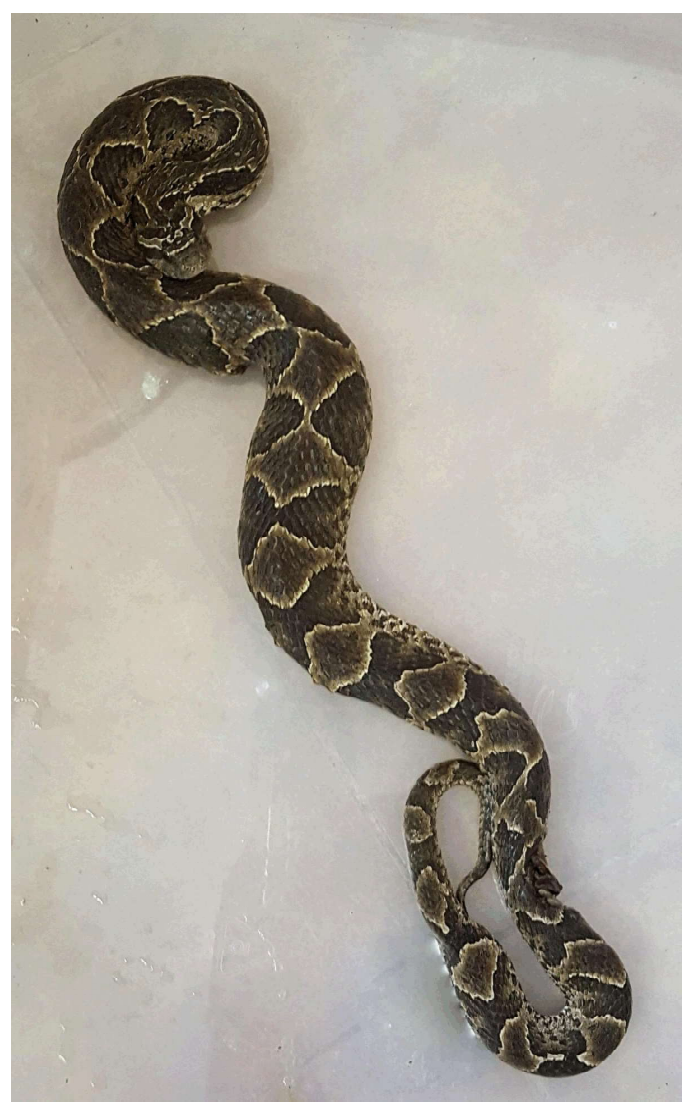

Figura 1. Paciente Xenodon merremii

dia y derecha del cuerpo. La piel presentaba una solución de continuidad costrosa, seca, no proliferativa, oscura, de $3 \mathrm{~cm}$ de largo y 1 cm de ancho (Figura 2a) y una lesión consistente en decoloración y pérdida de brillo de las escamas, $3 \mathrm{~cm}$ posterior a la cloaca (Figura $2 b$ ). El sistema musculoesquelético no evidenció alteraciones. La temperatura cloacal fue de $26^{\circ} \mathrm{C}$, la frecuencia cardíaca de 68 pulsaciones por minuto y la frecuencia respiratoria de 22 por minuto. Mucosa cloacal normal y mucosa bucal seca, pero de coloración normal.

Debido al grado de deshidratación y aletargamiento del animal, se realizó fluidoterapia con solución de Ringer Lactato $(20 \mathrm{ml} / \mathrm{kg} / 24 \mathrm{~h}$ por vía subcutánea), complementado con vitaminas del complejo B (10 mg/kg cada $24 \mathrm{~h}$ por vía subcutánea durante 5 días), ya que el reptil rehusaba alimentarse. Localmente se realizó la limpieza diaria de las lesiones utili- 

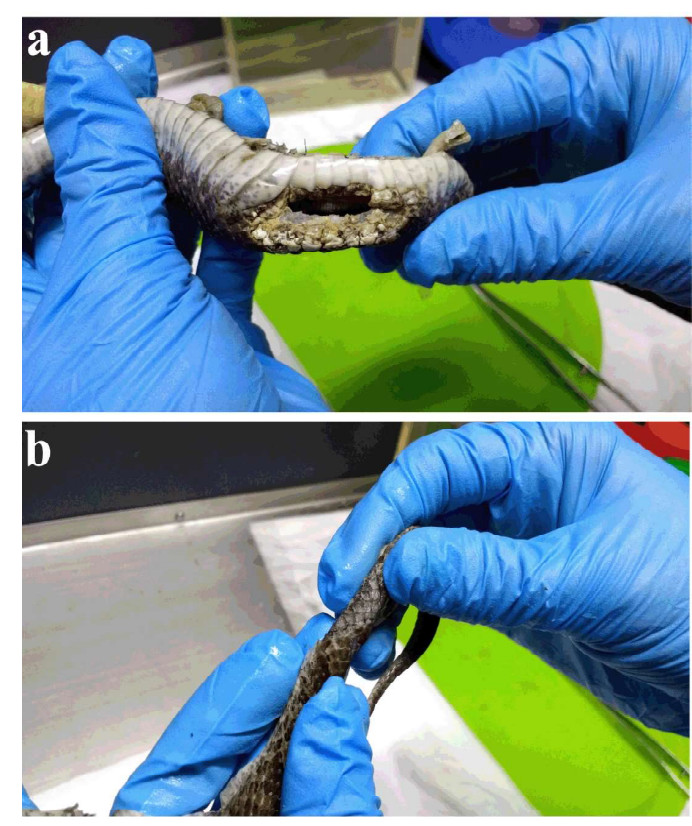

Figura 2. Lesiones en paciente adulto Xenodon merremii. a) Solución de continuidad costrosa en la piel del tercio medio del cuerpo. b) Decoloración y pérdida de brillo de las escamas

zando yodopovidona a concentración de $10 \%$. Para evitar infecciones agregadas se administró sulfadimetoxina $(90 \mathrm{mg} / \mathrm{kg}$ cada $24 \mathrm{~h}$ durante 5 días, por vía intramuscular en el tercio craneal del cuerpo). El reptil permaneció en cuarentena desde su llegada hasta su fallecimiento (16 días después de su arribo).

Debido al desenlace fatal del caso, se realizó la necropsia, la cual se inició con una inspección completa del tegumento, cavidad bucal, fosas nasales, ojos y cloaca. Luego se posicionó al ofidio en decúbito dorsal y se realizó una incisión longitudinal ventral siguiendo la línea alba, desde la cloaca hasta el espacio inter-mandibular. Se desplazó la piel hacia los lados, localizándose la membrana celómica, la cual fue inspeccionada y retirada. Se procedió luego a la extracción de las vísceras para su estudio macroscópico y microscópico, según la metodología descrita por Martínez Silvestre et al. (2001).
Se tomaron muestras de hígado, pulmón y piel, que fueron fijadas en formaldehído tamponado al $10 \%$ por $24 \mathrm{~h}$ y procesadas por técnicas histológicas convencionales para bloques parafinados. Los cortes se hicieron con micrótomo tipo Minot en un espesor de 5 micras, $y$ teñidos con hematoxilina-eosina $(\mathrm{H}-$ E) y metenamina de plata Grocott. Las observaciones se realizaron con microscopio óptico binocular (Primo Star, Carl Zeiss) con cámara digital (Axiocam ERc 5s, Carl Zeiss).

\section{Resultados}

Se hizo un diagnóstico presuntivo de EFS por las características de las lesiones presentadas, que luego fueron confirmadas con el análisis histopatológico.

El hígado, a la observación macroscópica, presentó focos blanquecinos y aplanados, de 1-2 mm de diámetro (Figura 3a), mientras que, a la microscopía óptica, el parénquima hepático presentó focos de tejido necrótico e infiltrado inflamatorio de células mononucleares entre los hepatocitos (Figura 3b). Las secciones coloreadas con tinción de metenamina de plata de Grocott mostraron elementos levaduriformes en los focos de necrosis. (Figura 3c). En contraste con el hígado, el pulmón no presentó alteraciones macroscópicas de relevancia, pero los cortes histológicos teñidos con H-E evidenciaron neumonía granulomatosa difusa (Figura 4a) y con la tinción de metenamina de plata de Grocott se evidenciaron elementos fúngicos consistentes en hifas septadas entre agregados de macrófagos y cúmulos de detritos celulares necróticos (Figura 4b).

En las lesiones de piel se evidenció una dermatitis necroulcerativa multifocal con edema dermo-epidérmico extenso e infiltrado inflamatorio (Figura 5). 

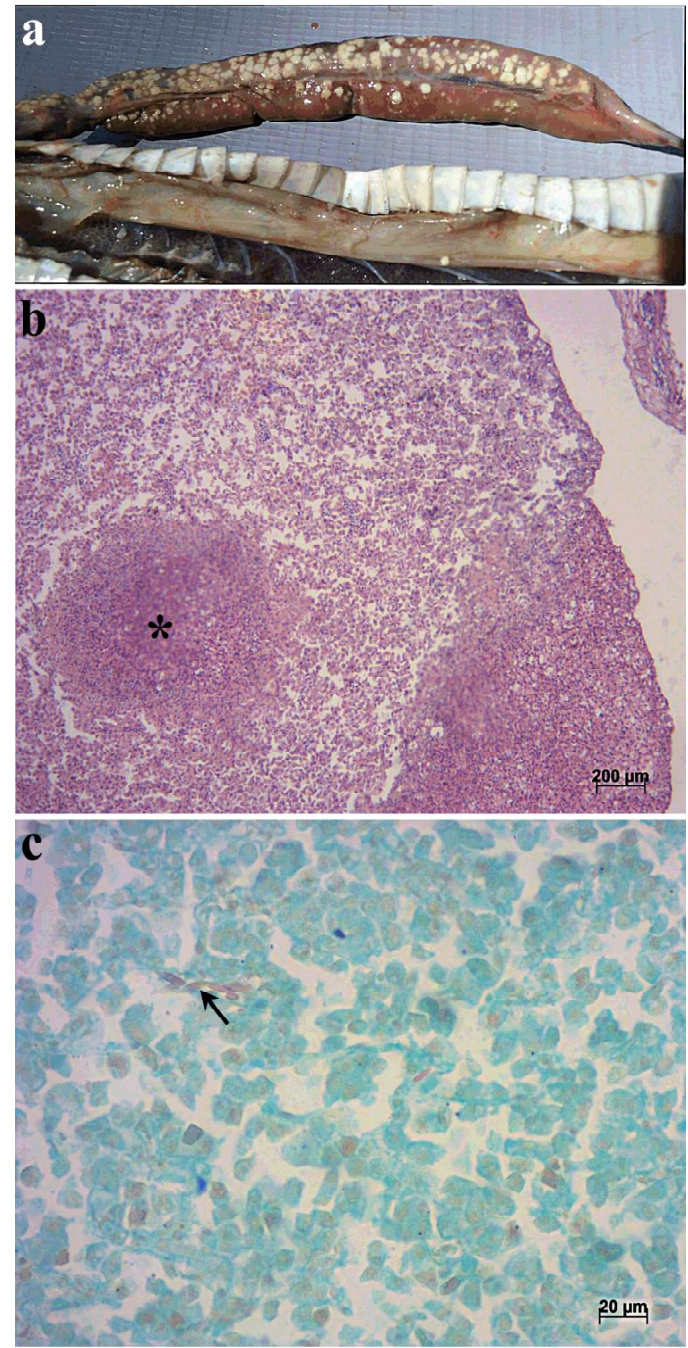

Figura 3. Lesiones en paciente adulto Xenodon merremii. a) Imagen macroscópica del hígado. Se observan focos blanquecinos, aplanados, en toda la superficie del órgano. b) Focos de tejido necrótico en el parénquima hepático acompañados de infiltrado inflamatorio (*). H-E. c) Se observan elementos levaduriformes en los focos de necrosis (flecha) del parénquima hepático. Tinción de Grocott

\section{Discusión}

Las lesiones costrosas obsrrvadas en la piel del espécimen de $X$. merremii son similares a las descritas por McBride et al. (2015)

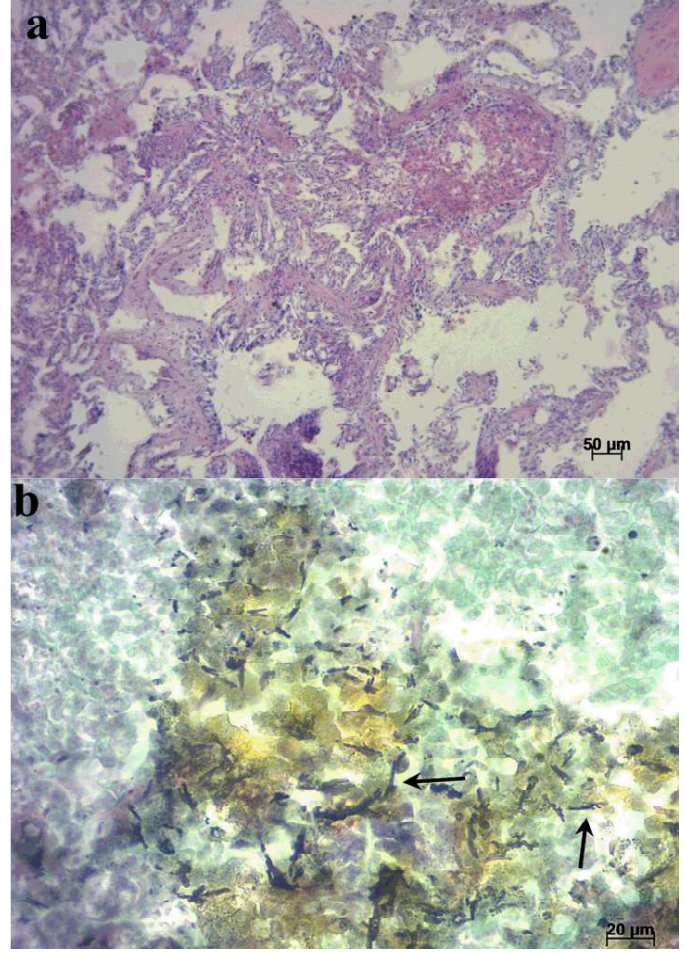

Figura 4. Corte histológico de pulmón de Xenodon merremii. a) Se observa neumonía granulomatosa acompañada de un infiltrado inflamatorio, predominantemente mononuclear. H-E. b) Nótese la presencia de hifas septadas (flechas) entre cúmulos de detritos celulares necróticos en pulmón. Tinción de metenamina de plata de Grocott

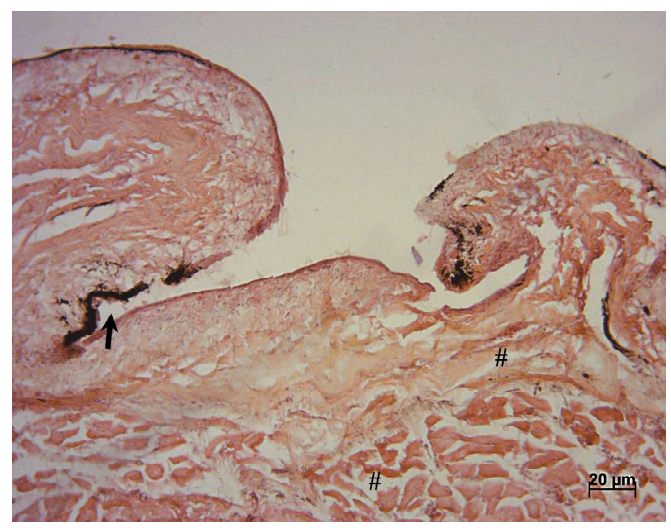

Figura 5. Corte histológico de piel de Xenodon merremii. Se observa dermatitis necroulcerativa (flecha) multifocal con edema dermo-epidérmico extenso (\#). H-E 
en los vipéridos Crotalus horridus, Sistrurus catenatus y Sistrurus miliarius; donde las lesiones faciales fueron causadas por $O$. ophiodiicola, principalmente a nivel de las fosetas nasolabiales. En el presente reporte, las lesiones se presentaron en una ubicación anatómica diferente, ya que se localizaron cercanas a la cloaca y en el tercio medio del cuerpo, además de presentarse una micosis sistémica.

Hasta hace unos años atrás, se creía que las lesiones en piel características de la EFS afectaban solamente la cabeza y el cuello de los ofidios, pero actualmente se sabe que pueden ser observadas en todo el cuerpo (Allender et al., 2015a), lo cual coincide con los hallazgos realizados en este trabajo. Por otro lado, las lesiones observadas a nivel de hígado guardan similitud con las reportados por Dolinski et al. (2014). Así mismo, las alteraciones micóticas observadas en pulmón son semejantes a las observadas por Vissiennon et al. (1999).

Según Allender et al. (2015a), la presencia de lesiones ulceradas y costrosas localizadas en la parte media del cuerpo de una serpiente es frecuente. Además, dicho autor hace referencia a la alta tasa de mortalidad por la EFS en vipéridos, indicando que la enfermedad ocurre generalmente luego del periodo de hibernación, posiblemente debido al ayuno donde su metabolismo ha disminuido, y donde se registran los recuentos leucocitarios más bajos (Jacobson, 2007). El caso aquí reportado ocurrió en septiembre, época en que finaliza la hibernación de esta especie (Carreira et al., 2005).

Los primeros hallazgos de O. ophiodiicola en serpientes Sistrurus miliarius, Thamnophis sp, Crotalus horridus y Sistrurus catenatus se reportaron en los estados de Florida, New Hampshire e Illinois, EEUU (Allender et al., 2015b), los cuales poseen climas similares (húmedos, calurosos y con abundantes precipitaciones) que constituyen ambientes propicios para el desarrollo del hongo. Estas características climáticas se asemejan a las del municipio de Riachuelo, lugar de donde provino la serpiente Xenodon merremii de este caso.

Varios autores hacen referencia a la presencia de esta enfermedad en colúbridos (Nichols et al., 1999; Vissiennon et al., 1999; Bertelsen et al., 2005; Rajeev et al., 2009; Dolinski et al., 2014); sin embargo, no se dispone de reportes de esta patología en Xenodon merremii, serpiente de amplia distribución en Sudamérica. Se concluye que el paciente presentó lesiones compatibles con EFS.

\section{Agradecimiento}

Los autores agradecen a la MV Winnie Merlo del Servicio de Histopatología del Hospital de Clínicas de la Facultad de Ciencias Veterinarias de la Universidad Nacional del Nordeste.

\section{Literatura Citada}

1. Allender MC, Dreslik M, Wylie S, Phillips C, Wylie DB, Maddox C, Delaney MA, Kinsel MJ. 2011. Chrysosporium sp infection in eastern massasauga rattlesnakes. Emerg Infect Dis 17: 2383-2384. doi: 10.3201/ eid1712.110240

2. Allender MC, Baker S, Willie D, Loper D, Dreslik MJ, Phillips CA, Maddox C, Driskell EA. 2015a. Development of snake fungal disease after experimental challenge with Ophidiomyces ophiodiicola in cottonmouths (Agkistrodon piscivorous). PloS One 10(10): e0140193. doi: 10. 1371/Journal. pone. 0140193

3. Allender MC, Bunick D, Dzhaman E, Burrus L, Maddox C. 2015b. Development and use of a real-time polymerase chain reaction assay for the detection of Ophidiomyces ophiodiicola in snakes. J Vet Diagn Invest 27: 217-220. doi: $10.1177 / 1040638715573983$ 
4. Allender MC, Raudabaugh DB, Gleason FH, Miller AN. 2015c. The natural history, ecology, and epidemiology of Ophidiomyces ophiodiicola and its potential impact on free-ranging snake populations. Fungal Ecol 17: 187-196. doi: 10.1016/j.funeco.2015.05.003

5. Allender MC. 2017. Ophidiomyces Project - Wildlife Epidemiology Lab College of Veterinary Medicine University of Illinois. [Internet]. Disponible en: http://vetmed.illinois.edu/wel/ ophidiomyces-project/

6. Bertelsen MF, Crawshaw GJ, Sigler L, Smith DA. 2005. Fatal cutaneous mycosis in tentacle snakes (Erpeton tentaculatum) caused by the Chrysosporium anamorph of Nannizziopsis vriesii. J Zoo Wild Med 36: 82-87. doi: 10.1638/04-020

7. Carreira S, Meneghel M, Achaval F. 2005. Reptiles de Uruguay. Montevideo, Uruguay: Facultad de Ciencias, Universidad de la República. 640 p.

8. Dolinski A, Allender M, Hsiao V, Maddox C. 2014. Systemic Ophidiomyces ophiodiicola infection in a freeranging plains garter snake (Thamnophis radix). J Herpetol Med Surg 24: 710. doi: 10.5818/1529-9651-24.1.7

9. Franklinos L, Lorch J, Bohuski E, Rodríguez-Ramos J, Wright $O$, Fitzpatrick L, Petrovan S, et al. 2017. Emerging fungal pathogen Ophidiomyces ophiodiicola in wild European snakes. Sci Rep 7(1): 3844. doi: 10.1038/ s41598-017-03352-1

10. Giraudo AR. 2004. Serpientes de la selva paranaense y del Chaco húmedo. $2^{\circ} \mathrm{ed}$. Buenos Aires, Argentina: Ed. LOLA. 328 p.
11. Jacobson ER. 2007. Infectious diseases and pathology of reptiles. Color atlas and text. CRC Press. $736 \mathrm{p}$.

12. Martínez-Silvestre A, Brotóns NJ, Ramis A. 2001. Patología de reptiles. Canis et Felis 49: 21-70.

13. McBride MP, Wojick KB, Georoff TA, Kimbro J, Garner MM, Wang X, et al. 2015. Ophidiomyces ophiodiicola dermatitis in eight free-ranging timber rattlesnakes (Crotalus horridus) from Massachusetts. J Zoo Wild Med 46: 8694. doi: 10.1638/2012-0248R2.1

14. Nichols DK, Weyant R, Lamirande EW, Sigler Mason RT. 1999. Fatal mycotic dermatitis in captive brown tree snakes (Boiga irregularis). J Zoo Wild Med 30: 111-118.

15. Rajeev S, Sutton DA, Wickes B, Miller DL, Giri D, Van Meter M, Thompson $\boldsymbol{E H}$, et al. 2009. Isolation and characterization of a new fungal species, Chrysosporium ophiodiicola, from a mycotic granuloma of a black rat snake (Elaphe obsoleta obsoleta). J Clin Microbiol 47: 1264-1268. doi: 10.1128/ JCM.01751-08

16. Sleeman J. 2013. Snake fungal disease in the United States. Nat Wildl Health Center Wildl Health Bull 2: 1-3.

17. Varela N. 2002. Evaluación clínica de reptiles. Bol GEAS 3: 1-11.

18. Vissiennon T, Schüppel KF, Ullrich E, Kuijpers AFA. 1999. Case report. A disseminated infection due to Chrysosporium queenslandicum in a garter snake (Thamnophis). Mycoses 42: 107-110. doi: 10.1046/j.14390507.1999.00409.x 